\title{
Use of Optical Fibres for Multi-parameter Monitoring in Electrical AC Machines
}

\author{
David Hind, Chris Gerada, Michael Galea, James Borg \\ Bartolo \\ Power Electronics, Machines and Control (PEMC) Group \\ University of Nottingham \\ Nottingham, UK \\ chris.gerada@nottingham.ac.uk
}

\author{
Matthias Fabian, Tong Sun, Kenneth T. V. Grattan \\ City, University of London \\ 10 Northampton Square \\ London, UK \\ Matthias.Fabian.1@city.ac.uk
}

\begin{abstract}
This paper describes a new approach to multiparameter monitoring for electrical $\mathrm{AC}$ machines. It is demonstrated that speed, torque and temperature can be measured using optical fibres incorporating sensors in the form of fibre Bragg gratings (FBGs) distributed around the machine. One fibre can incorporate several FBGs and hence provide several measurements. Experimental results showing speed, torque, direction of rotation, stator housing vibration and temperature measured using the FBG method are presented and validated against measurements obtained from conventional sensors. The results show that the optical fibre based approach allows multiple parameters to be monitored accurately and simultaneously with only a fraction of the usual monitoring equipment required. Another advantage of the proposed method is the EMI immunity naturally provided by optical solutions. The presented measurement technique can also offer a new alternative approach to sensorless control.
\end{abstract}

Keywords-Optical Fibre; Fibre Bragg Grating (FBG); multi parameter monitoring; Health monitoring; Sensorless.

\section{INTRODUCTION}

Parameter monitoring is an important part of any motor drive system for several reasons including control, model validation, diagnostics, drive health monitoring and component lifetime prediction purposes. Conventional multiparameter monitoring systems often include a number of individual subsystems (sensors and interrogation equipment); each dedicated to monitoring a particular parameter. Such systems have high cost and space requirements associated with them. Additionally, in the case of motor speed and position detection, mechanical position sensors (either encoders or resolvers) are often considered the weak link in any motor drive, particularly when operating in demanding environments. Eliminating the position sensor and estimating the rotor position by alternative means could offer several benefits including increased drive reliability, robustness, reduced physical package size and also reduced manufacturing inputs in terms of cost, construction time and calibration.

This paper describes a new approach to multiparameter monitoring; the new technique relies on the use of optical fibre sensors, specifically fibre Bragg grating (FBG) sensors, strategically placed around the machine. One fibre can incorporate many sensors therefore allowing several measurements to be made using a single fibre. It is possible to measure several parameters including speed, position, torque, temperature and vibration. Speed and position estimates can be used for closed loop control therefore removing the need for a mechanical position sensor. Using this technique does not require any modification to the inverters' electrical output signals (as with some conventional sensorless approaches) and placement of the fibres around the machine has no impact on overall physical package size. Fibres can be used to measure multiple quantities; obtaining such information usually requires multiple and independent instrumentation and interrogation equipment systems.

There are many failure modes that can affect electric machines and these failure modes tend to have indicators related to them, for example bearing failures can be associated with a particular vibration harmonic and magnet demagnetization in PM machines is brought about by excessive rotor temperature. The use of optical fibres for multi-parameter monitoring enables greater protection and reliability to be introduced into real time drive systems by tracking failure indicator parameters, which can help to maintain the running of critical systems and minimize downtime.

This work also presents an exciting new opportunity for adding to the field of sensorless control. Numerous approaches are described in literature; mathematical modelling techniques such as the Model Based Reference System (MRAS) [1-3] and observer based methods [4-6] offer excellent performance at high speed but fail at low speed where the feedback variables (e.g. Back EMF) become small and highly susceptible to noise. Saliency tracking methods aim to track local magnetic saturations in the machine and can operate across a large speed range including zero speed. However, these approaches require a high frequency (HF) signal (in addition to the fundamental waveform) to be applied to the machine. The HF waveform can be added either as a signal to the vector control demands prior to calculating the PWM pulses and timings (so called high frequency injection) [7-11], or by manipulating slightly the inverter switching edges themselves [12-15]. The addition of HF content to the fundamental waveform brings undesirable consequences such as torque ripple, audible noise, vibration, increased current 
total harmonic distortion (THD) and voltage limitations on the inverter. The method presented in this work is able to track the fundamental wave and could hence be used to identify rotor position. No additional signals need be added to the inverter waveform and operation at zero and low speed is possible.

\section{PRINCIPLE}

Various optical fibre sensing techniques have previously been used for parameter monitoring in motor drive applications. The parameters monitored include temperature [16], end-winding vibration [17], stator housing vibration [18], thermal effects [19], torque [20] and stator wave and speed [21]. FBG's were applied in [22] to measure voltage graduation of end windings with the aim of detecting any damage to an anti-corona coating applied to end windings. The principle involved using FBGs to determine voltage by measuring surface strain in response to an applied electric field. In [23] an optical fibre sensor in the form of an in-line fibre etalon (ILFE) was used as a vibrometer to detect the voltage unbalance in induction machines. While in [24] a simple fibre optic accelerometer was developed to monitor low amplitude, low frequency vibrations on large rotating machinery.

This paper aims to expand the work in [21] by monitoring multiple parameters simultaneously in real time through a single interrogation unit. Identification of the direction of rotation of the rotor is also introduced. The principle utilised in this work relies on fibre Bragg gratings formed within optical fibres acting as notch filters in transmission and reflecting light at the so-called Bragg wavelength. An in-depth description of the phenomenon can be found in literature [25]. Thermal and mechanical variations (strain, bending) affect the Bragg wavelength and are widely reported. Through the correct placement of such sensors within the electrical machine, this variation can be detected and correlated to motor parameters.

\section{EXPERIMENTAL SETUP}

In order to demonstrate the new multi-parameter monitoring system a back to back motor drive setup was commissioned. This arrangement featured a prototype $2 \mathrm{~kW}$ permanent magnet (PM) motor as the machine under test (MUT). The motor, designed for aerospace applications, was instrumented with optical fibres to allow fibre based measurements to be made. Loading of the MUT was provided by an Oswald asynchronous machine coupled to the MUT via an inline Magtrol TMHS 311/111 torque sensor. The torque sensor was used to give an independent measurement of torque and speed for validation purposes. The machines were each powered via off-the-shelf, M700 converters from Control Techniques, sharing a common DC-link . An Active Front End (AFE) was used to maintain the DC link and supply the power-loss component.

A K-type thermocouple was placed on the stator end winding of the MUT to provide validation thermal data. Finally, a Micron Optics SM130 interrogator sampling at 2 $\mathrm{kHz}$ was used to capture the optical signals. Processing of the received data was then performed in a custom LabVIEW

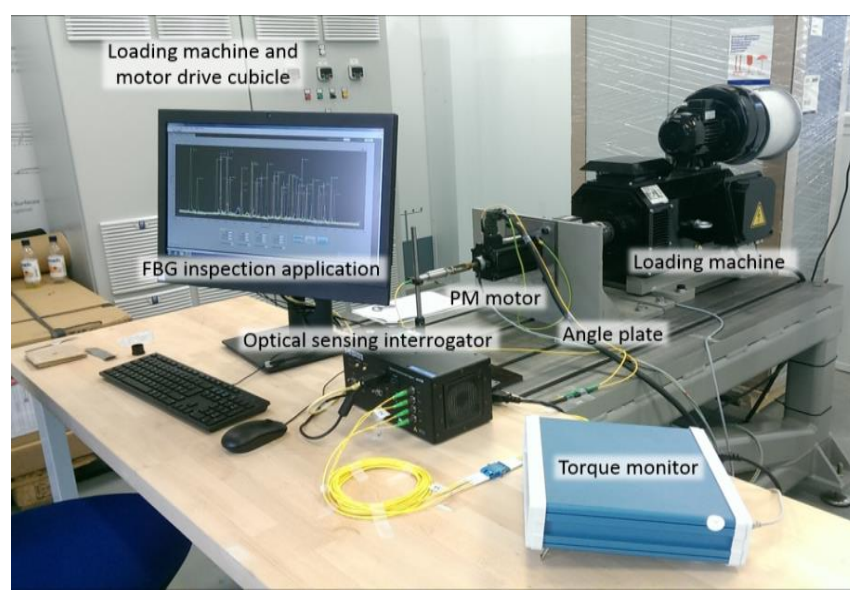

Fig. 1 The experimental setup used to test the FBG method

based application. The experimental setup is photographed in Fig. 1.

\section{A. MUT Stator Instrumentation}

Three fibres were installed onto the stator of the MUT. Each fibre had a total of twelve FBG sensors. Two fibres were placed along the length of the machine in between the stator teeth in an "up and down" alternate slot fashion as shown in Fig. 2 (a). The FBGs of these fibres (two per stator slot) were used to measure temperature and obtain a thermal profile of the stator. One fibre was used to measure position and speed by circumferentially mounting the FBG sensors between stator teeth as illustrated in Fig. 2 (b). Strain due to mechanical displacement of the stator teeth (due to the fundamental wave and it's harmonics) can be measured and used to provide speed and position information [21].

\section{B. MUT Rotor Instrumentation}

Measurement of torque complicates matters as this measurement must be made on the surface of the rotor shaft. A differential wavelength approach which uses two FBGs attached to the rotor shaft at an angle of $\pm 45^{\circ}$ with respect to the spinning axis was used [20]. When the FBGs are placed in this configuration the difference between the two FBG reflection peak wavelengths provides a torque measurement while their mid-point is an indicator for the temperature at that location. If the fibres are not placed at exactly $\pm 45^{\circ}$ during

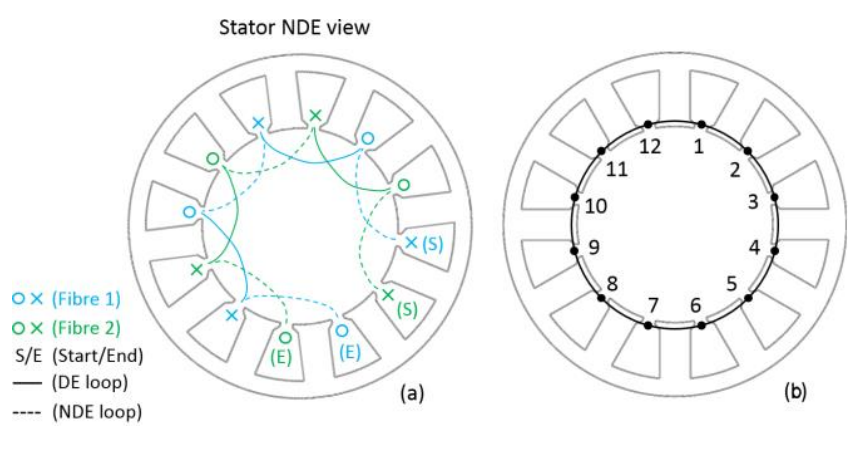

Fig. 2 Stator FBG arrangements: (a) shows the looped path of the stator temperature monitoring FBGs. FBGs were mounted in-between stator teeth with two FBGs per slot. (b) Shows the mounting arrangement of the position/speed sensing FBGs on the end of the stator teeth 

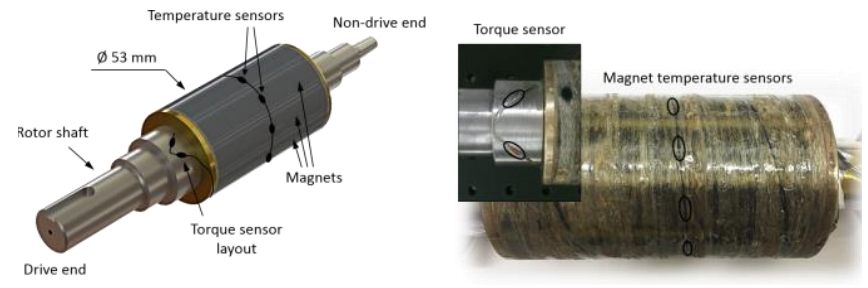

Fig. 3 Rotor FBG instrumentation showing the positioning of the torque sensing FBGs and the rotor temperature FBGs

instrumentation then some temperature dependence will be introduced which can be compensated for by continuously monitoring their mid-point wavelength. As measurement of the torque only requires the use of two FBGs and each fibre contains twelve, the remaining ten FBGs could be distributed around the rotor surface and used to measure rotor temperature. Rotor temperature can be valuable information, particularly when using permanent magnet machines where there is a risk of exceeding the safe-operating temperature of the magnets hence irreversibly degrading motor performance. Fig. 3 illustrates the rotor fibre installation for torque and temperature measurement. Some arrangement is required to get the optical signal from the fibre mounted on the rotating shaft to the stationary interrogator. In this work a commercial fibre-optic rotary joint was used along with a standard high speed flexible coupling to cater for a small shaft misalignment. This allowed the signal to pass from the stationary interrogator to the rotating shaft. This rotating joint represents a mechanical limitation of the experimental setup in this work since the shaft alignment and mechanical support of the joint in this implementation was not sufficient to support high speed operation (>1000RPM). It is thought that an improved optical interface between the stationary and rotating parts could be designed in order to reduce this limitation.

\section{EXPERIMENTAL RESULTS}

This section presents results for torque, speed, direction of rotation, stator housing vibration and temperature (both rotor and stator) estimation. In each case (excluding rotor temperature and direction of rotation) the estimated parameters are compared with validation data obtained through standard sensing techniques.

\section{A. Speed}

Fig. 4. shows the estimated speed measured from the FBG approach plotted against the measured speed from a standard encoder mounted within the Magtrol TMHS 311/111 torque sensor. The results show the response to a number of step changes in the speed demand, as well as a ramped change (between 35 and 65 seconds). The results show that the estimated speed closely matches the real measured speed. Currently the processing applied to the response of the fibres involves using a Fourier transform to obtain a frequency domain response and then tracking the second harmonic of the stator frequency. While this works well, it requires a large window time, particularly at low speed. Work is ongoing to improve the data extraction element of the implementation;

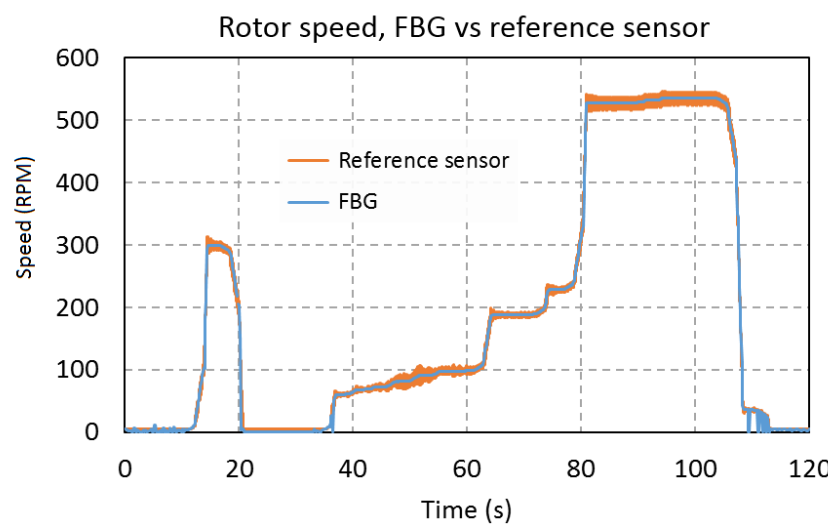

Fig. 4 Speed transients with speed measured using FBG approach and encoder for reference

however the principle has been shown to work well across the speed range including operation at zero and low speed.

\section{B. Stator Housing Vibration}

Stator housing vibration was also measured using the FBG approach and compared with a result obtained using a commercial vibration analyser. The results illustrated in Fig. 5 show a reasonable agreement, with the FBG approach being able to correctly identify the dominant component in the frequency response.

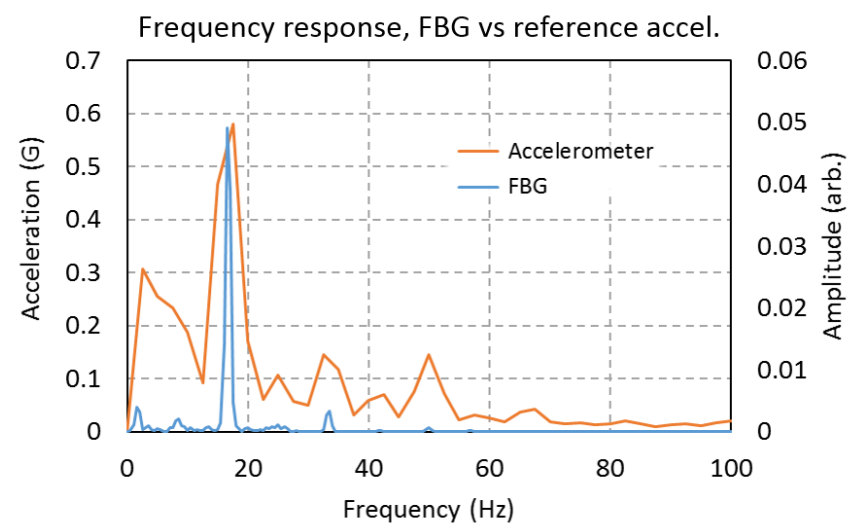

Fig. 5 Stator housing vibration measured using FBG and an independent vibration analyser

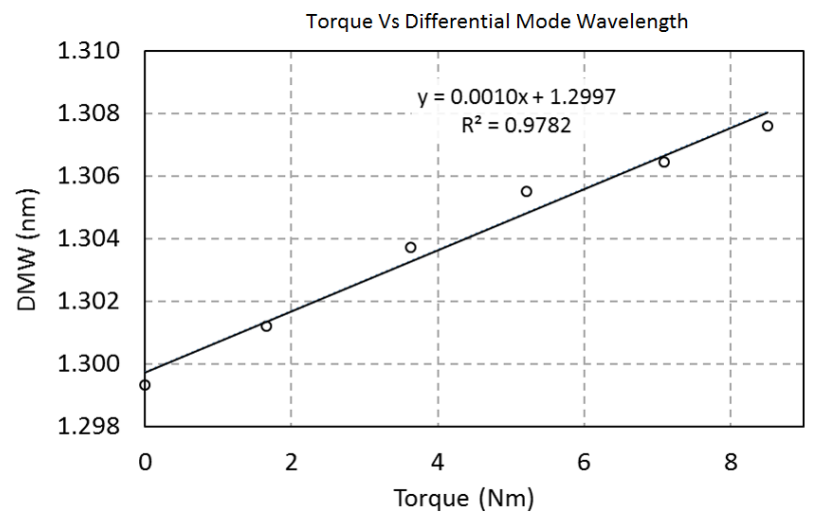

Fig. 6 Relationship between shaft torque and differential mode wavelength measured from the FBG system 


\section{Torque}

In the case of torque measurement by the FBG system, a parameter called the differential mode wavelength (DMW) is measured. The DMW is the distance between the reflection peaks of the two FBGs placed at $\pm 45^{\circ}$ on the rotor shaft surface and can be directly related to the torque experienced by the rotor shaft. Fig. 6 shows the relationship between differential mode wavelength (DMW) and the torque measured by the Magtrol 311/111 torque sensor. The results show a reasonably linear relationship, inferring that the torque can be accurately estimated through the DMW.

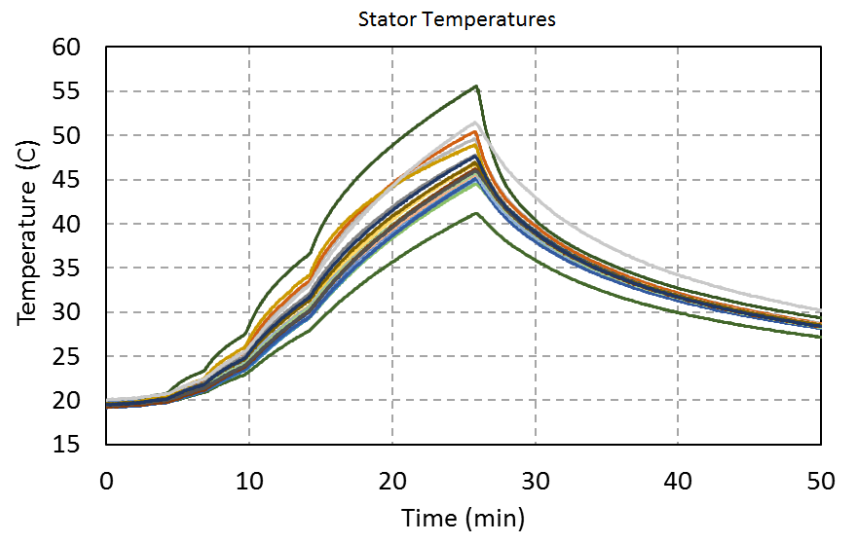

(a)

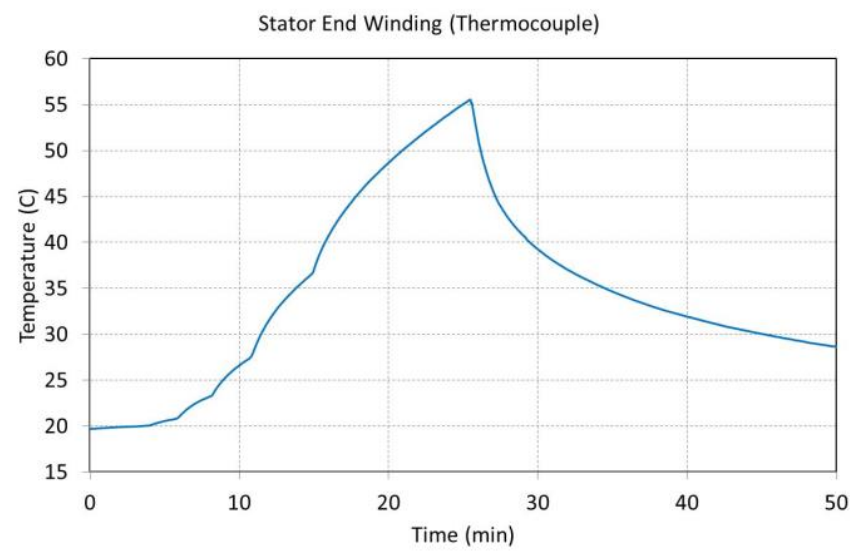

(b)

Fig. 7 Stator Thermal data acquired from the FBGs (a) and thermocouple located on stator end winding (b)

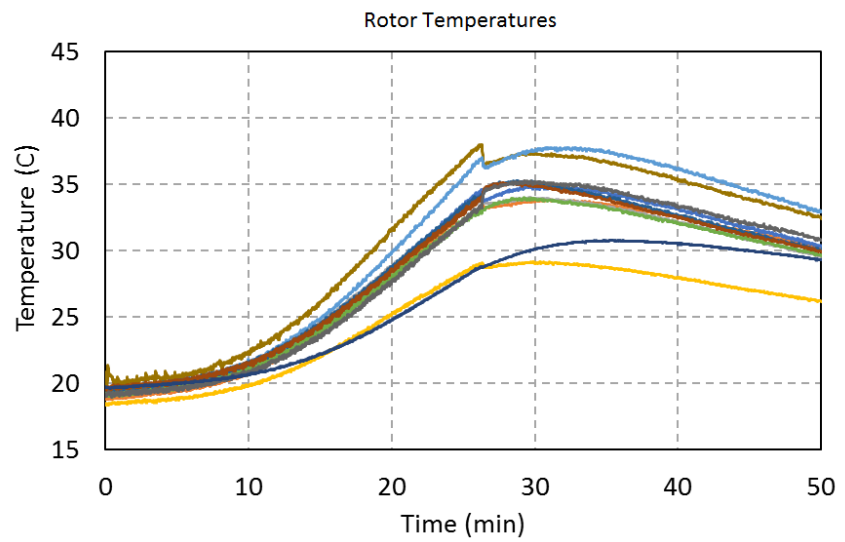

Fig. 8 Rotor thermal data acquired from the FBGs

\section{Temperature}

Fig. 7 (a) shows the 24 stator winding temperature measurements from the optical fibres. For validation, an independent temperature measurement taken at the stator end winding is shown in Fig 7 (b). It can be seen that the thermocouple measurement and the optical fibre measurements are a close match. The stator winding temperatures are higher than the rotor measurements due to the increased losses experienced by the stator. It is thought that the stator end winding temperatures are slightly higher due to the concentrated nature of the end winding losses. Another consideration is the lack of stator material between the end windings to dissipate heat. Fig. 8 shows the ten rotor temperature measurements.

\section{E. Direction of Shaft Rotation}

The responses of the FBGs mounted circumferentially on the end of the stator teeth can be used to identify the direction of shaft rotation as well as position. Fig. 9 (a) and (b) show the time domain responses of three consecutively placed FBGs for positive and negative spinning directions respectively. It can clearly be seen that the phase order of the FBGs is reversed with a change in spinning direction and hence this can easily be used to determine the shafts direction of rotation.

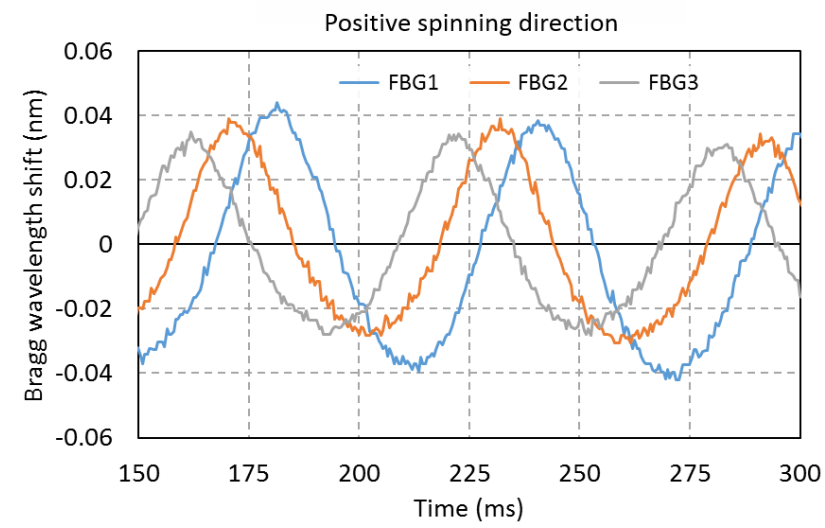

(a)

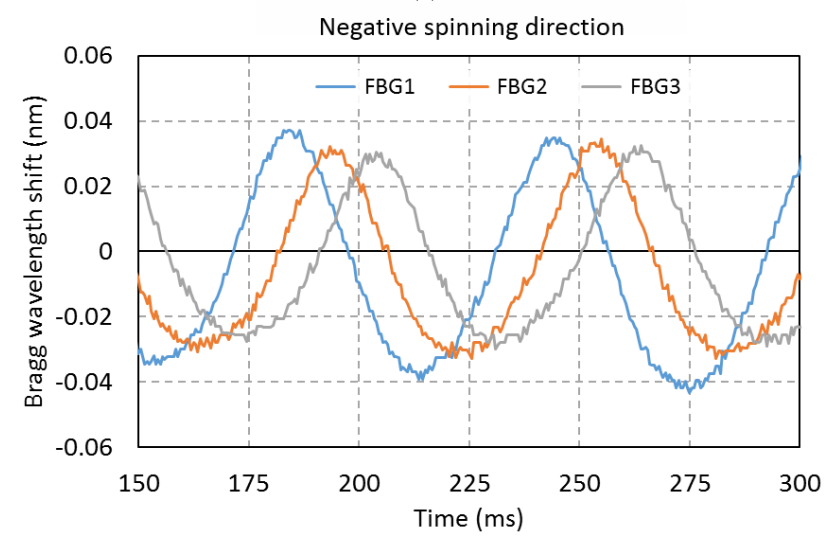

(b)

Fig. 9 Consecutive (circumferentially mounted) FBG responses for positive (a) and negative (b) rotor shaft spinning directions 


\section{CONCLUSIONS}

The results presented in this paper show that it is possible to use Fibre Bragg Sensors to accurately monitor speed, torque, temperature, shaft rotation direction and stator housing vibration. A single fibre can include many FBGs and can therefore easily be used to obtain a thermal profile of the machine. The approach presented here does not necessitate bulky sensors and requires only one interrogation unit leading to a very compact solution in terms of physical space requirements. The optical fibres can be routed to machines in remote, compact or challenging environments without concern and the transmission of information is immune to EMC issues. The speed and position information present an opportunity for a new alternative approach to sensorless control of the machine.

\section{ACKNOWLEDGMENT}

The authors wish to acknowledge the funding provided by the EU Clean Sky Initiative for this investigation.

\section{REFERENCES}

[1] C. Schauder, "Adaptive speed identification for vector control of induction motors without rotational transducers," Industry Applications, IEEE Transactions on, vol. 28, pp. 1054-1061, 1992.

[2] P. L. Jansen and R. D. Lorenz, "Accuracy limitations of velocity and flux estimation in direct field oriented induction machines," in Power Electronics and Applications, 1993., Fifth European Conference on, 1993, pp. 312-318 vol.4.

[3] P. Fang-Zheng and T. Fukao, "Robust speed identification for speedsensorless vector control of induction motors," Industry Applications, IEEE Transactions on, vol. 30, pp. 1234-1240, 1994.

[4] M. Tursini, R. Petrella, and F. Parasiliti, "Adaptive sliding-mode observer for speed-sensorless control of induction motors," Industry Applications, IEEE Transactions on, vol. 36, pp. 1380-1387, 2000.

[5] K. Kubota, K. Matsuse, and T. Nakano, "DSP-based speed adaptive flux observer of induction motor," Industry Applications, IEEE Transactions on, vol. 29, pp. 344-348, 1993.

[6] J. Maes and J. A. Melkebeek, "Speed-sensorless direct torque control of induction motors using an adaptive flux observer," Industry Applications, IEEE Transactions on, vol. 36, pp. 778-785, 2000.

[7] P. L. Jansen and R. D. Lorenz, "Transducerless position and velocity estimation in induction and salient AC machines," Industry Applications, IEEE Transactions on, vol. 31, pp. 240-247, 1995.

[8] Q. Gao, G. Asher, and M. Sumner, "Sensorless Position and Speed Control of Induction Motors Using High-Frequency Injection and Without Offline Precommissioning," IEEE Transactions on Industrial Electronics, vol. 54, pp. 2474-2481, 2007.

[9] H. Jung-Ik and S. Seung-Ki, "Sensorless field-orientation control of an induction machine by high-frequency signal injection," Industry Applications, IEEE Transactions on, vol. 35, pp. 45-51, 1999.

[10] A. Consoli, G. Scarcella, and A. Testa, "Sensorless control of PM synchronous motors at zero speed," in Industry Applications Conference, 1999. Thirty-Fourth IAS Annual Meeting. Conference Record of the 1999 IEEE, 1999, pp. 1033-1040 vol.2.
[11] Y. D. Yoon, S. K. Sul, S. Morimoto, and K. Ide, "High-Bandwidth Sensorless Algorithm for AC Machines Based on Square-Wave-Type Voltage Injection," IEEE Transactions on Industry Applications, vol. 47, pp. 1361-1370, 2011.

[12] M. Schroedl, "Sensorless control of AC machines at low speed and standstill based on the "INFORM" method," in Industry Applications Conference, 1996. Thirty-First IAS Annual Meeting, IAS '96., Conference Record of the 1996 IEEE, 1996, pp. 270-277 vol.1.

[13] Q. Gao, G. M. Asher, M. Sumner, and P. Makys, "Sensorless Control of Induction Machines, including Zero Frequency using only Fundamental PWM Excitation," in IEEE Industrial Electronics, IECON 2006 - 32nd Annual Conference on, 2006, pp. 793-798.

[14] J. Holtz and P. Hangwen, "Elimination of saturation effects in sensorless position controlled induction motors," in Industry Applications Conference, 2002. 37th IAS Annual Meeting. Conference Record of the, 2002, pp. 1695-1702 vol.3.

[15] T. M. Wolbank and J. Machl, "A modified PWM scheme in order to obtain spatial information of AC machines without mechanical sensor," in Applied Power Electronics Conference and Exposition, 2002. APEC 2002. Seventeenth Annual IEEE, 2002, pp. 310-315 vol.1.

[16] A. A. Boiarski and J. P. Kurmer, "Fiber optic sensors for temperature and strain monitoring in motors and generators," Electric Power Research Institute, TR-101950-V2, 2487-02, Final Report 1997.

[17] P. Kung, L. Wang, and M. I. Comanici, "Stator end winding vibration and temperature rise monitoring," in 2011 Electrical Insulation Conference (EIC). 2011, pp. 10-14.

[18] J. M. Corres, J. Bravo, F. J. Arregui, and I. R. Matias, "Unbalance and harmonics detection in induction motors using an optical fiber sensor," IEEE Sensors Journal, vol. 6, pp. 605-612, 2006.

[19] K. d. M. Sousa, A. A. Hafner, H. J. Kalinowski, and J. C. C. d. Silva, "Determination of Temperature Dynamics and Mechanical and Stator Losses Relationships in a Three-Phase Induction Motor Using Fiber Bragg Grating Sensors," IEEE Sensors Journal, vol. 12, pp. 3054-3061, 2012.

[20] P. L. Swart, A. A. Chtcherbakov, and A. J. Van Wyk, "Dual Bragg grating sensor for concurrent torsion and temperature measurement," Meas. Sci. Technol. 17(5) pp. 1057-1064 2006.

[21] M. Fabian, J. Borg Bartolo, M. Ams, C. Gerada, T. Sun, and K. T. V. Grattan, "Vibration measurement of electrical machines using integrated fibre Bragg gratings," 2015, pp. 963417-963417-4.

[22] F. Marignetti, E. de Santis, S. Avino, G. Tomassi, A. Giorgini, P. Malara, P. De Natale and G. Gagliardi, "Fiber Bragg Grating Sensor for Electric Field Measurement in the End Windings of High-Voltage Electric Machines," in IEEE Transactions on Industrial Electronics, vol. 63, no. 5, pp. 2796-2802, May 2016.

[23] J. M. Corres, J. Bravo, F. J. Arregui and I. R. Matias, "Unbalance and harmonics detection in induction motors using an optical fiber sensor," in IEEE Sensors Journal, vol. 6, no. 3, pp. 605-612, June 2006.

[24] J. M. Lopez-Hignera, M. A. Morante and A. Cobo, "Simple lowfrequency optical fiber accelerometer with large rotating machine monitoring applications," in Journal of Lightwave Technology, vol. 15, no. 7, pp. 1120-1130, Jul 1997.

[25] Y. J. Rao, "In-fibre bragg grating sensors," Meas. Sci. Technol. 8(4),, pp. 355-375 1997. 